\title{
High-resolution Imaging of Spherulitic Structures in PLLA and PDLA Solvent-cast Films
}

\author{
Manon Guivier ${ }^{1}$, Wen Qian ${ }^{1}$, Joseph A. Turner ${ }^{1}$ and Antonella Esposito ${ }^{2}$ \\ ${ }^{1}$ University of Nebraska-Lincoln, Lincoln, Nebraska, United States, ${ }^{2}$ Université Rouen-Normandie, Saint- \\ Etienne-du-Rouvray, Haute-Normandie, France
}

The stereocomplexation of polylactic acid (PLA) for blends of poly(L-lactic acid) (PLLA) and poly(Dlactic acid) (PDLA) has been studied for several decades with the perspective of enhanced thermal and mechanical properties. The melting temperature of sterecomplex PLA obtained in equimolar PLLA/PDLA blends is $50{ }^{\circ} \mathrm{C}$ higher thanure PLLA and pure PDLA [1], with higher tensile strength [2]. In order to understand the improved properties of PLLA/PDLA blends, it is first necessary to investigate the structure of pure PLLA and pure PDLA individually. In this article, nanoscale infrared and contact resonance atomic force microscopy (IR-AFM and CR-AFM) are used to obtain a chemical (IR) and mechanical (frequency stiffness) mapping of the spherulitic structures grown in pure PLLA and pure PDLA solventcast films at the nanoscale (less than $30 \mathrm{~nm}$ ). The films were cast from a solution with an initial concentration of $33.33 \mathrm{mg} / \mathrm{mL}$, and with a final thickness of $300 \mathrm{~nm}$. The solution was prepared by dissolving the PLLA and PDLA pellets into chloroform and then poured on a glass slide, dried at room temperature $\left(22^{\circ} \mathrm{C}\right)$ for 48 hours and crystallized at $130{ }^{\circ} \mathrm{C}$ for $10-12$ minutes.

Fig. 1 (a) and (b) show the topography images of entire PDLA and PLLA spherulites over a scan area of $50 \mu \mathrm{m} \times 50 \mu \mathrm{m}$. By positioning the AFM probe at a specific location, localized infrared (IR) spectra were obtained with nanoscale resolution for both PLLA and PDLA spherulitic structures (Fig. 1 (c)). The probe tip radius is approximately $35 \mathrm{~nm}$, which is far below the conventional optical diffraction limit. Three primary absorption peaks $\left(928 \mathrm{~cm}^{-1}, 1450 \mathrm{~cm}^{-1}\right.$ and $\left.1790 \mathrm{~cm}^{-1}\right)$ are observed that can be ascribed to the $\mathrm{C}-\mathrm{C}$ stretching, to the $\mathrm{CH}_{3}$ bending and to the $\mathrm{C}=\mathrm{O}$ stretching, respectively [3]. The magnified topography image of the PDLA spherulite within a scan area of $3 \mu \mathrm{m} \times 3 \mu \mathrm{m}$ is shown in Fig. 1 (d). In order to distinguish the crystalline from the amorphous areas, chemical mapping was used with a fixed wavenumber of $928 \mathrm{~cm}^{-1}$ as well as a phase lock loop (PLL) frequency mapping $(171.47 \pm 50 \mathrm{kHz})$, as shown in Fig. 1 (e) and Fig. 1 (f). In the chemical mapping, the red areas respond more than the blue areas in terms of C-C stretching, which is related to a different arrangement of the polymer chains. In the frequency mapping, the red areas correspond with a stiffer response, whereas the blue areas are less stiff. The combination of these three images is consistent with the expectation of having crystalline lamellae surrounded by amorphous thin layers inside the spherulite. The yellow regions of Fig. 1 (e) and the red regions of Fig. 1 (f) represent the stiffer phase and the blue regions on Fig. 1 (e) and Fig. 1 (f) represent the softer phase, which are expected to be the crystalline and amorphous phases, respectively. A similar interpretation can be made for the spherulitic structures grown of the PLLA film (Fig. 1 (g), (h) and (i)). However, the presence of lamellae inside PLLA spherulites is unclear and the arrangement of the polymer chains is definitely less organized in comparison with the PDLA spherulite. An attempt to distinguish the crystalline phase from the amorphous phase in PDLA spherulite was also made using CR-AFM frequency mapping (Fig. 2 (a) and (b)). The resonance frequency was locked at a value of $113.06 \mathrm{kHz} \pm 30 \mathrm{kHz}$ to collect the information for frequency mapping over a scan area of $3 \mu \mathrm{m} \times 3 \mu \mathrm{m}$ considering only the first symmetric mode. Both organized (stiffer) regions and less organized (softer) regions are observed, which may correspond with the crystalline and amorphous regions of PDLA spherulites. The correlation is made by comparing Fig. 2 (b) with Fig. 1 (f). The layered structure, with a clear contrast between the crystalline phase and the mobile amorphous, was obtained in both cases by stiffness mapping. Similar analyses were 
performed for the PLLA spherulite, with the resonance frequency locked at $116.61 \mathrm{kHz} \pm 30 \mathrm{kHz}$. However, the crystalline phase and the surrounding regions cannot be clearly distinguished (Fig. 2 (d)).

These preliminary results show that the combination of localized IR-AFM and CR-AFM allows resolving the localized spherulitic structures grown in PLLA and PDLA solvent-cast films, and that PLLA spherulites appear much less organized than PDLA spherulites grown under the same conditions [4].
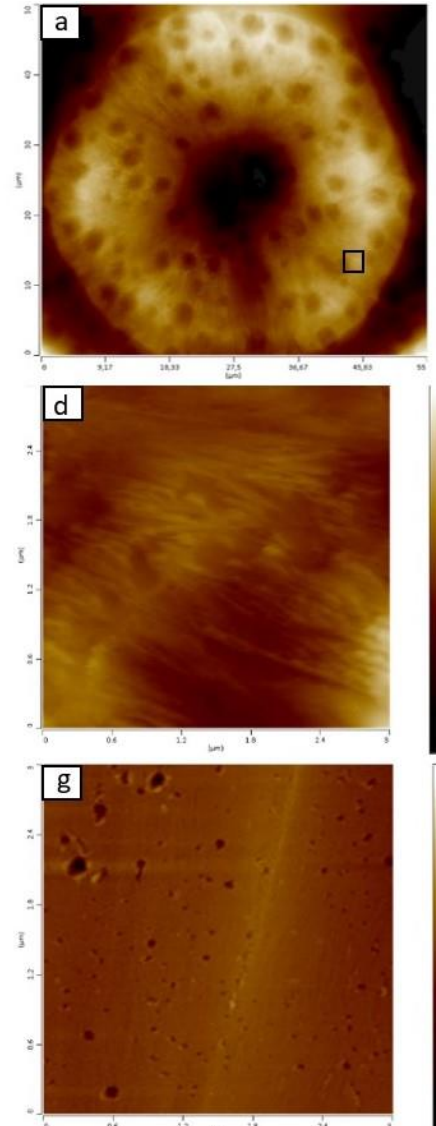

Figure 1. Topography images of entire (a) PDLA and (b) PLLA spherulites; (c) localized IR spectra of PDLA (orange) and PLLA (blue) spherulites; (d) magnified topography image of a PDLA spherulite (scanning area $3 \mu \mathrm{m} \times 3 \mu \mathrm{m}$ ); (e) IR map of Figure 1 (d) at a fixed wavenumber of $928 \mathrm{~cm}-1$; (f) PLL frequency map of Figure (d) at $171.47 \pm 50 \mathrm{kHz} ;(\mathrm{g})$ magnified topography image , (h) chemical IR map and (i) PLL frequency map of a region of a PLLA spherulite.
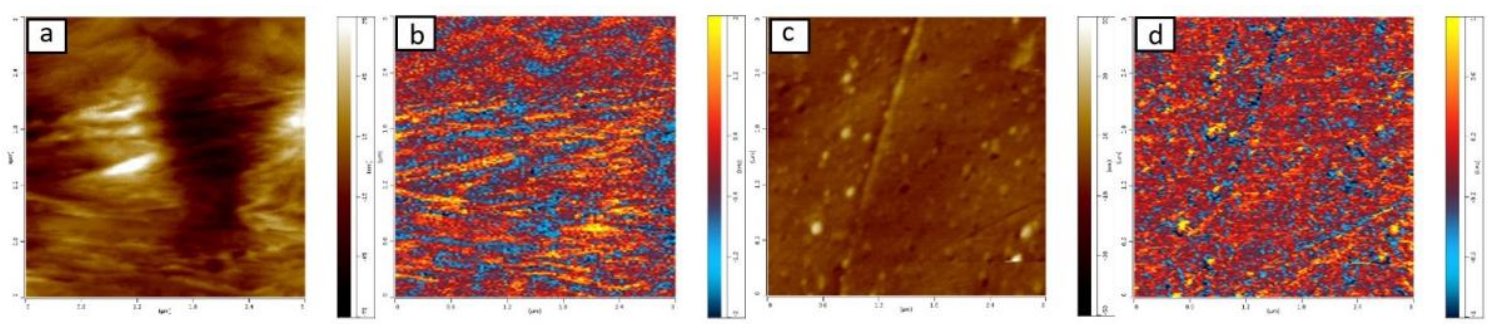

Figure 2. CR-AFM resonance frequency maps of the first symmetric mode (scanning area $3 \mu \mathrm{m} \times 3$ $\mu \mathrm{m}$ ) (a) topography image of a PDLA spherulite; (b) frequency map of Figure 2 (a) at $113.06 \pm 30 \mathrm{kHz}$; (c) topography image of a local region of a PLLA spherulite; (d) frequency map of Figure 2 (c) at 116.61 $\pm 30 \mathrm{kHz}$. 


\section{References}

[1] Okihara, T., et al. J. Macromol. Sci. Part B (1991), 30 (1-2), 119-140.

[2] Tsuji, H., and Y. Ikada. Polymer 40.24 (1999): 66996708.

[3] Thangaraju, Elakkiya, et al. Fibers and Polymers 13.7 (2012): 823-830

[4] This research was supported in part by the funds from the Nebraska Research Initiative. 